\title{
Social support and hippocampal volume are negatively associated in adults with previous experience of childhood maltreatment
}

\author{
Katharina Förster, PhD*; Lorenz Danzer*; Ronny Redlich, PhD, PhD; Nils Opel, MD; \\ Dominik Grotegerd, PhD; Elisabeth J. Leehr, PhD; Katharina Dohm, PhD; \\ Verena Enneking, MSc; Susanne Meinert, MSc; Janik Goltermann, MSc; \\ Hannah Lemke, MSc; Lena Waltemate, MSc; Katharina Thiel, MSc; Katja Behnert; \\ Katharina Brosch, MSc; Frederike Stein, MA; Tina Meller, PhD; Kai Ringwald, MSc; \\ Simon Schmitt, MSc; Olaf Steinsträter, PhD; Andreas Jansen, PhD; Axel Krug, PhD; \\ Igor Nenadic, MD; Tilo Kircher, MD; Tim Hahn, PhD; Harald Kugel, PhD; \\ Walter Heindel, MD; Jonathan Repple, MD; Udo Dannlowski, MD, PhD
}

\begin{abstract}
Background: Childhood maltreatment has been associated with reduced hippocampal volume in healthy individuals, whereas social support, a protective factor, has been positively associated with hippocampal volumes. In this study, we investigated how social support is associated with hippocampal volume in healthy people with previous experience of childhood maltreatment. Methods: We separated a sample of 446 healthy participants into 2 groups using the Childhood Trauma Questionnaire: 265 people without maltreatment and 181 people with maltreatment. We measured perceived social support using a short version of the Social Support Questionnaire. We examined hippocampal volume using automated segmentation (Freesurfer). We conducted a social support $\times$ group analysis of covariance on hippocampal volumes controlling for age, sex, total intracranial volume, site and verbal intelligence. Results: Our analysis revealed significantly lower left hippocampal volume in people with maltreatment (left $F_{1,432}=5.686, p=0.018$; right $F_{1,433}=3.371, p=0.07$ ), but no main effect of social support emerged. However, we did find a significant social support $\times$ group interaction for left hippocampal volume (left $F_{1,432}=5.712, p=0.017$; right $F_{1,433}=3.480, p=0.06$ ). In people without maltreatment, we observed a trend toward a positive association between social support and hippocampal volume. In contrast, social support was negatively associated with hippocampal volume in people with maltreatment. Limitations: Because of the correlative nature of our study, we could not infer causal relationships between social support, maltreatment and hippocampal volume. Conclusion: Our results point to a complex dynamic between environmental risk, protective factors and brain structure - in line with previous evidence - suggesting a detrimental effect of maltreatment on hippocampal development.
\end{abstract}

\section{Introduction}

Childhood maltreatment is an adverse social experience and is a risk factor for many mental disorders. ${ }^{1}$ It is also associated with neuroendocrine dysregulation (e.g., hypercortisolism) and alterations in brain structure., ${ }^{2,3}$ In particular, hippocampal volume has repeatedly been shown to be negatively associated with childhood maltreatment in healthy people, as well as in people with major depressive disorder (MDD). ${ }^{4-6}$ As well, evidence from longitudinal studies suggests that these brain structural alterations mediate the influence of childhood maltreatment on the development and clinical outcomes of major depression. ${ }^{78}$ In addition to changes in hippocampal structure, people who have experienced maltreatment show differences in limbic activity ${ }^{9}$ and connectivity ${ }^{10}$ to other areas involved in emotion regulation, such as the medial prefrontal cortex. It has been repeatedly suggested that these "limbic scars" imply that people are more vulnerable to developing mental health disorders after being exposed to childhood maltreatment. ${ }^{5}$

Correspondence to: U. Dannlowski, Department of Psychiatry, University of Münster, Albert-Schweitzer-Campus 1, G A9, 48149 Münster, Germany; dannlow@uni-muenster.de

*These authors contributed equally to this paper.

Submitted Aug. 14, 2020; Revised Nov. 24, 2020; Accepted Dec. 31, 2020

DOI: 10.1503/jpn.200162 
Unlike research into the neurobiological correlates of risk factors (such as childhood maltreatment), research focused on brain structures associated with protective factors is scarce. ${ }^{11}$ Protective factors are external and internal mechanisms for coping with stressful situations without losing level of function. ${ }^{12}$ One important protective factor against the development of psychiatric illnesses (including MDD) is social support. ${ }^{13}$ Social support can be measured as perceived social support, in the intensity of the feeling of being loved, protected, accepted and respected ${ }^{14}$ using self-report questionnaires with brief statements such as "I can easily find someone to look after my home when I'm not there."15 Social support can also be measured in terms of social network size and received social support. ${ }^{16}$ High levels of social support are associated with a higher quality of life, the expression of positive emotions and reduced psychological anguish. ${ }^{17} \mathrm{~A}$ high level of social support in people with maltreatment is associated with a lower risk of developing mental disorders, more perceived psychological satisfaction and better personal development. ${ }^{18}$

In addition to directly improving psychological well-being, social support may reduce the impact of negative life events and act as a buffer against the effects of adversity - this is also referred to as the "buffer hypothesis."13,16 This buffer might also be represented in altered structural brain development. Here, the hippocampus is of particular interest because of its high density of glucocorticoid receptors and increased sensitivity toward stress, ${ }^{8,19}$ as well as its varying susceptibility to alterations of nurturance during maturation. The hippocampus increases in size until age 2 years, and then neurogenesis decreases rapidly after this period. ${ }^{20,21}$ However, up to the age of 8 years, hippocampal volume is still positively associated with parental nurturance, ${ }^{22}$ indicating that reduced nurturance during this period (i.e., maltreatment) disturbs maturation of the hippocampus, and increased nurturance may compensate for previous experiences of adversity. After this period, nurturance is not associated with hippocampal volume, strengthening the hypothesis that parental support is highly relevant to hippocampal maturation up to the age of 8 , but not afterward. This hypothesis was further substantiated by a recent longitudinal neuroimaging study that investigated the effect of social support of a primary caregiver during different time periods in childhood. ${ }^{23-26}$ During preschool, caregiver support predicts a later increase of childhood hippocampal volume ${ }^{25,26}$ and mediates the negative relationship between hippocampal volume and stressful events. ${ }^{24}$ In school-aged children, however, higher caregiver support was associated with hippocampal volume increases only if they reported a low frequency of adverse life events. ${ }^{23}$ Thus, the time period during which nurturance and support are received affects how they affect hippocampal volume.

During adulthood, social support has been associated with increased grey matter volumes in areas such as the posterior cingulate cortex, the lingual cortex, the left occipital lobe and the cuneus, ${ }^{27}$ as well as the amygdala. ${ }^{11}$ In a study of brain functional alterations, participants who reported higher social support showed no association between trait anxiety and amygdala reactivity to threat-related cues. ${ }^{28}$ This finding also underlines the idea that social support acts as a buffer in the face of adversity. In summary, previous studies have revealed that social support is a protective factor in the development of individual resilience in general and against the stressor of childhood maltreatment. ${ }^{18,29}$ However, how social support is associated with the hippocampus during adulthood in people with exposure to childhood maltreatment remains elusive. In the present study, we investigated the interaction of childhood maltreatment and social support on hippocampal brain structure. We hypothesized that participants with previous experience of childhood maltreatment would show lower hippocampal volumes than participants without experience of childhood maltreatment. We also hypothesized that social support would be positively associated with hippocampal volume. Finally, because the effect of childhood maltreatment on hippocampal volume is detrimental and occurs during a sensitive period of hippocampal development, ${ }^{20}$ and because studies point toward a reduced susceptibility of the hippocampus toward nurturance after preschool, 22,23,30 we hypothesized that social support would show a different association with hippocampal volume in people with maltreatment than in those without maltreatment.

\section{Methods}

\section{Participants}

All participants took part in the Marburg-Münster Affective Disorders Cohort Study (MACS). The MACS is a longitudinal cohort study investigating patients with MDD, bipolar disorder, schizoaffective disorder and schizophrenia, as well as healthy participants at genetic and/or environmental risk (e.g., childhood maltreatment) and control participants with no environmental or genetic risk $(n=2500)$. Details of recruiting and data processing have been described elsewhere. ${ }^{31}$ Our study was approved by the local institutional ethics committees of Marburg and Münster, and all participants provided written informed consent before participation. Participants were financially compensated for taking part in the study. All procedures contributing to this work complied with the ethical standards of relevant national and institutional committees on human experimentation and with the Helsinki Declaration of 1975 (revised 2008). All participants included in the present analysis showed no evidence of previous psychiatric disorders according to DSM-IV criteria based on a structured clinical interview (SCID- ${ }^{32}$ ). Additional exclusion criteria were neurologic abnormalities or previous traumatic head injury; chronic medical disease; or MRI contraindications.

A total sample of 446 participants was included. The sample comprised 181 participants who had a score greater than or equal to the cut-off for childhood maltreatment ${ }^{33}$ on at least 1 subscale of the Childhood Trauma Questionnaire (CTQ), ${ }^{34}$ referred to in this paper as "people with maltreatment." From a large sample of participants who scored below the cut-off on all CTQ subscales in the MACS, we included 
265 participants matched to the people with maltreatment based on age, sex, perceived social support, depression score and verbal intelligence - referred to in this paper as "people without maltreatment."

\section{Data collection and ethical approval}

Study data are part of the MACS (DFG FOR2107) and represent original work. Data collection was approved by the local ethics committee of the medical faculty of the University of Münster and the medical faculty in Marburg. All participants provided informed consent before participating in the studies.

The FOR2107 cohort project was approved by the Ethics Committees of the Medical Faculties, University of Marburg (AZ: 07/14) and University of Münster (AZ: 2014-422-b-S).

\section{Behavioural measures}

We measured social support using the 22-item version of the Social Support Questionnaire. ${ }^{35,36}$ The questionnaire measures a person's subjective anticipation of receiving social support in case of need and assesses the social resources in the person's environment using brief statements about situations of everyday life. It shows high internal consistency (Cronbach's $\alpha=0.85-0.93) .{ }^{15}$ The reliability in our sample was moderate to high (Cronbach's $\alpha=0.81$ ). We measured the intensity of childhood maltreatment using the German version of the CTQ. ${ }^{34,37,38}$ The questionnaire measures childhood maltreatment with high internal consistency (Cronbach's $\alpha=0.79$ 0.94) using 5 subscales and their respective cut-off scores: ${ }^{33}$ physical abuse (cut-off 8), emotional abuse (cut-off 10), emotional neglect (cut-off 15), sexual abuse (cut-off 8) and physical neglect (cut-off 8). We assessed subclinical depressive symptoms using the Beck Depression Inventory. ${ }^{39}$ We also administered the German multiple-choice vocabulary test the MWT-B. ${ }^{40}$ The MWT-B is used to indicate verbal intelligence, although it shows only medium correlations with general intelligence (e.g., as measured by the Wechsler Adult Intelligence Scale). ${ }^{41}$ For descriptive statistics, we used a direct translation of the MWT-B to IQ points; ${ }^{40}$ for analyses, we used the raw MWT-B score. The MWT-B overestimates IQ by approximately 16 points, ${ }^{41}$ but IQ points were not interpreted in this study; they were used only as descriptive statistics. Therefore, the effect of the overestimation was negligible.

\section{Structural MRI}

Data were collected as part of the MACS at 2 sites. ${ }^{42}$ In Münster, data were collected using a 3 T Siemens PRISMA (20-channel head matrix Rx-coil) using 3D $T_{1}$-weighted magnetization-prepared rapid acquisition gradient echo (MPRAGE) sequence (repetition time $1900 \mathrm{~ms}$; echo time $2.28 \mathrm{~ms}$; inversion time $900 \mathrm{~ms}$; flip angle $8^{\circ}$; sagittal slices 192; slice gap $0 \mathrm{~mm}$; voxel size $1 \times 1 \times 1 \mathrm{~mm}^{3}$ ). In Marburg, data were collected using a 3 T Siemens Magnetom Trio Tim syngo MR B17 (12-channel head matrix Rx-coil) using a 3D $T_{1}$-weighted MPRAGE sequence (repetition time $1900 \mathrm{~ms}$; echo time $2.26 \mathrm{~ms}$; inversion time $=900 \mathrm{~ms}$; flip angle $9^{\circ}$; sagittal slices 176; slice gap $0.5 \mathrm{~mm}$; voxel size $\left.1 \times 1 \times 1 \mathrm{~mm}^{3}\right)$. In Marburg, a body-coil change occurred during data collection.

All structural images were preprocessed using the subcortical segmentation stream of Freesurfer (version 5.3) with default parameters. ${ }^{42}$ Based on standardized quality check protocols provided by the ENIGMA consortium (http:// enigma.ini.usc.edu/protocols/imagingprotocols), we used visual inspection to check our sample for artifacts (e.g., caused via movement) and segmentation quality. We analyzed the volumes of the left and right hippocampus based on the Desikan-Killiany atlas. ${ }^{43}$ We excluded 2 participants from analysis of the right hippocampal volume and 3 participants from analysis of the left hippocampal volume after visual inspection because of poor segmentation.

\section{Statistical analyses}

We analyzed the preprocessed Freesurfer data using SPSS 25 (IBM). We conducted a social support $\times$ group analysis of covariance controlling for age, sex, total intracranial volume, verbal intelligence, site and exchange of the body coil at the Marburg study site for the left and right hippocampus separately, based on our a priori hypotheses related to hippocampal volume. We applied Bonferroni correction in our regionof-interest analysis to correct for multiple comparisons of the left and right hippocampus, reducing our statistical significance threshold to $\alpha=0.025$. Despite a lack of specific hypotheses, we provided further exploratory analyses related to the interplay between childhood maltreatment and social support on other subcortical volumes (Appendix 1, available at jpn.ca/200162-a1).

\section{Results}

Characteristics of the participants are shown in Table 1.

The analysis of covariance yielded a main effect of group, driven by significantly lower left hippocampal volumes in people with maltreatment compared to people without maltreatment (left $F_{1,432}=5.686, p=0.018, \eta_{\mathrm{p}}^{2}=0.013$; right $F_{1,433}=$ $3.371, p=0.07, \eta_{\mathrm{p}}{ }^{2}=0.008$; Figure 1$)$. We found no significant main effect of social support (left $p=0.99$; right $p=0.76$ ). However, a significant social support $\times$ group interaction for hippocampal volume did emerge (left $F_{1,432}=5.712, p=0.017$, $\eta_{\mathrm{p}}{ }^{2}=0.013$; right $F_{1,433}=3.480, p=0.06, \eta_{\mathrm{p}}{ }^{2}=0.008$; Figure 2). Results for the subscales of the CTQ and how they interacted with social support can be found in Appendix 1.

The post hoc analysis revealed a negative association between hippocampal volume and social support in people with maltreatment (left $r=-0.169, p=0.013$; right $r=-0.112$, $p=0.07)$, in contrast to a tendency toward a positive association between hippocampal volume and social support in people without maltreatment (left $r=0.091, p=0.07$; right $r=$ $0.092, p=0.07)$.

\section{Discussion}

The present study demonstrates a moderating role for the protective factor social support in the relationship between 
Table 1: Participant characteristics

\begin{tabular}{|c|c|c|c|c|}
\hline Characteristic & $\begin{array}{l}\text { People without } \\
\text { maltreatment }^{\star} \\
\quad(n=265)\end{array}$ & $\begin{array}{l}\text { People with } \\
\text { maltreatment } \\
\quad(n=181)\end{array}$ & $\begin{array}{l}\text { Total sample* } \\
\quad(n=446)\end{array}$ & $p$ value \\
\hline \multicolumn{5}{|l|}{ Demographic characteristics } \\
\hline Age & $35.51 \pm 12.56$ & $35.67 \pm 13.08$ & $35.57 \pm 12.76$ & 0.90 \\
\hline$\%$ Female & 58.9 & 63.5 & 60.8 & $0.32 \ddagger$ \\
\hline $\mathrm{IQ}$ & $115.95 \pm 14.15$ & $114.10 \pm 13.53$ & $115.20 \pm 13.92$ & 0.17 \\
\hline Beck Depression Inventory score & $4.35 \pm 4.15$ & $5.06 \pm 4.82$ & $4.64 \pm 4.44$ & 0.10 \\
\hline Social support scale score & $4.43 \pm 0.50$ & $4.37 \pm 0.63$ & $4.40 \pm 0.56$ & 0.24 \\
\hline \multicolumn{5}{|l|}{ Childhood Trauma Questionnaire } \\
\hline Total score & $29.49 \pm 3.49(25,40)$ & $41.28 \pm 10.17(28,77)$ & $34.29 \pm 9.10(25,77)$ & $<0.001$ \\
\hline Emotional abuse score & $6.13 \pm 1.21(5,9)$ & $9.51 \pm 4.01(5,24)$ & $7.51 \pm 3.19(5,24)$ & $<0.001$ \\
\hline Physical abuse score & $5.23 \pm 0.55(5,7)$ & $6.45 \pm 2.48(5,18)$ & $5.73 \pm 1.74(5,18)$ & $<0.001$ \\
\hline Sexual abuse score & $5.01 \pm 0.14(5,7)$ & $5.69 \pm 2.03(5,20)$ & $5.29 \pm 1.34(5,20)$ & $<0.001$ \\
\hline Emotional neglect score & $7.64 \pm 2.29(5,14)$ & $11.34 \pm 4.57(5,25)$ & $9.15 \pm 3.86(5,25)$ & $<0.001$ \\
\hline Physical neglect score & $5.47 \pm 0.70(5,7)$ & $8.29 \pm 2.10(5,16)$ & $6.61 \pm 2.00(5,16)$ & $<0.001$ \\
\hline \multicolumn{5}{|c|}{$\begin{array}{l}\text { ANOVA }=\text { analysis of variance. } \\
\text { *Unless otherwise indicated, values are mean } \pm \text { standard deviation (minimum, maximum) of the sample. In people without maltreatment, no Childhood Trauma } \\
\text { Questionnaire subscale was above the cut-off; in people with maltreatment, at least } 1 \text { subscale was above the cut-off. } \\
\text { †Unless otherwise indicated, } p \text { values were derived from univariate ANOVA (uncorrected), not adjusted. } \\
\ddagger p \text { value derived from a } \chi^{2} \text { test (uncorrected). }\end{array}$} \\
\hline
\end{tabular}

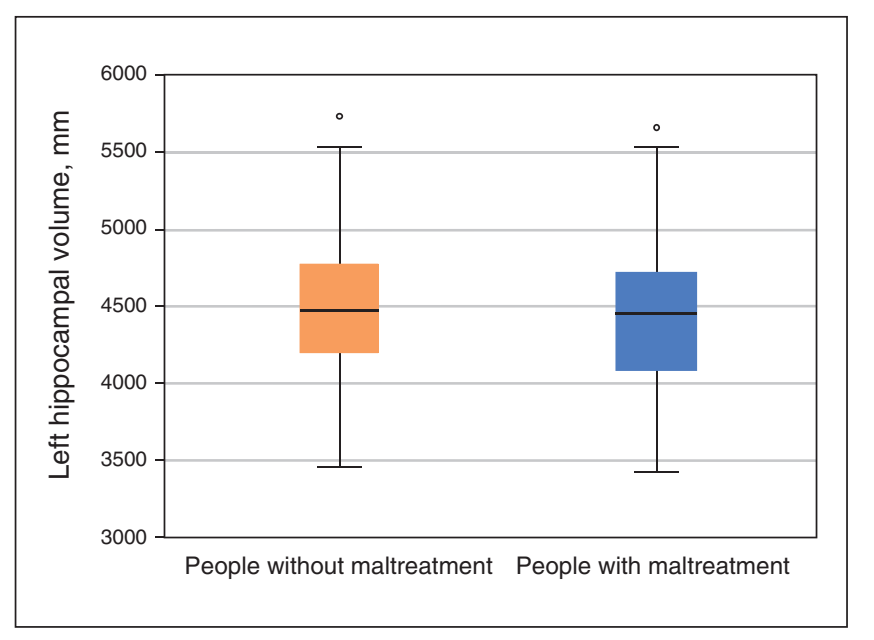

Fig. 1: Boxplots depicting predicted volumes of the left hippocampus in our univariate social support $\times$ group analysis of covariance, controlling for site, scanner, total intracranial volume, age, sex and verbal intelligence. We measured hippocampal volume using the automated segmentation software Freesurfer, comparing people without maltreatment to people with maltreatment, as defined by the Childhood Trauma Questionnaire. Participants with a score above the cut-off on at least 1 subscale of the questionnaire were categorized as people with maltreatment; all others were categorized as people without maltreatment. Points above the boxplots indicate values above the 75th percentile.

childhood maltreatment and hippocampal grey matter. More precisely, our findings revealed a trend toward a positive association between social support and hippocampal volume in healthy people without childhood maltreatment, but a negative association in people with childhood maltreatment. This novel result generates new hypotheses for the role of social support as a protective factor, given the detrimental effects of childhood maltreatment on hippocampal volume as described in our previous studies. ${ }^{4,5}$

In line with our hypothesis and several previous studies and meta-analyses, ${ }^{3,5,44}$ our results show that people with maltreatment exhibit lower left hippocampal volume than people without maltreatment. Moreover, smaller hippocampal volume is a frequent neuroimaging finding in patients with psychiatric disorders such as $\mathrm{MDD}^{45}$ and posttraumatic stress disorder, ${ }^{46}$ but also in healthy controls with increased risk of developing depression. ${ }^{8}$ These findings lead to the consideration of lower hippocampal volume as a transdiagnostic risk factor for mental disorders, rather than as a feature of them. ${ }^{47}$ Further, smaller hippocampal volume could mediate the relationship between childhood maltreatment and the development of psychopathology. ${ }^{8}$ The hippocampus is a structure with high expression of glucocorticoid receptors, and thus is susceptible to the detrimental effects of stress. ${ }^{19,48}$ Because of its rapid development, the brain is most plastic during childhood. ${ }^{30,49}$ Structural brain alterations because of childhood maltreatment in pivotal episodes of brain development might be masked by differences in the development of brain regions and synaptic organization. ${ }^{50,51}$ Lower hippocampal volume might result from decreased growth in early key periods and/or later increased loss in adolescence and adulthood. ${ }^{50}$ It thus seems plausible that alterations in hippocampal volume result from childhood maltreatment. Further, childhood maltreatment might generally lead to heightened vulnerability by increasing susceptibility to stress in later life, with lower hippocampal volume as a brain structural representation of this vulnerability. ${ }^{5}$ A stressor in later life such as trauma or chronic stress would then trigger the onset of a mental disorder. ${ }^{5}$ This possibility is underlined by research showing that hippocampal volume further decreases with the number and duration of stressful life events, such as hospitalizations, in patients with 


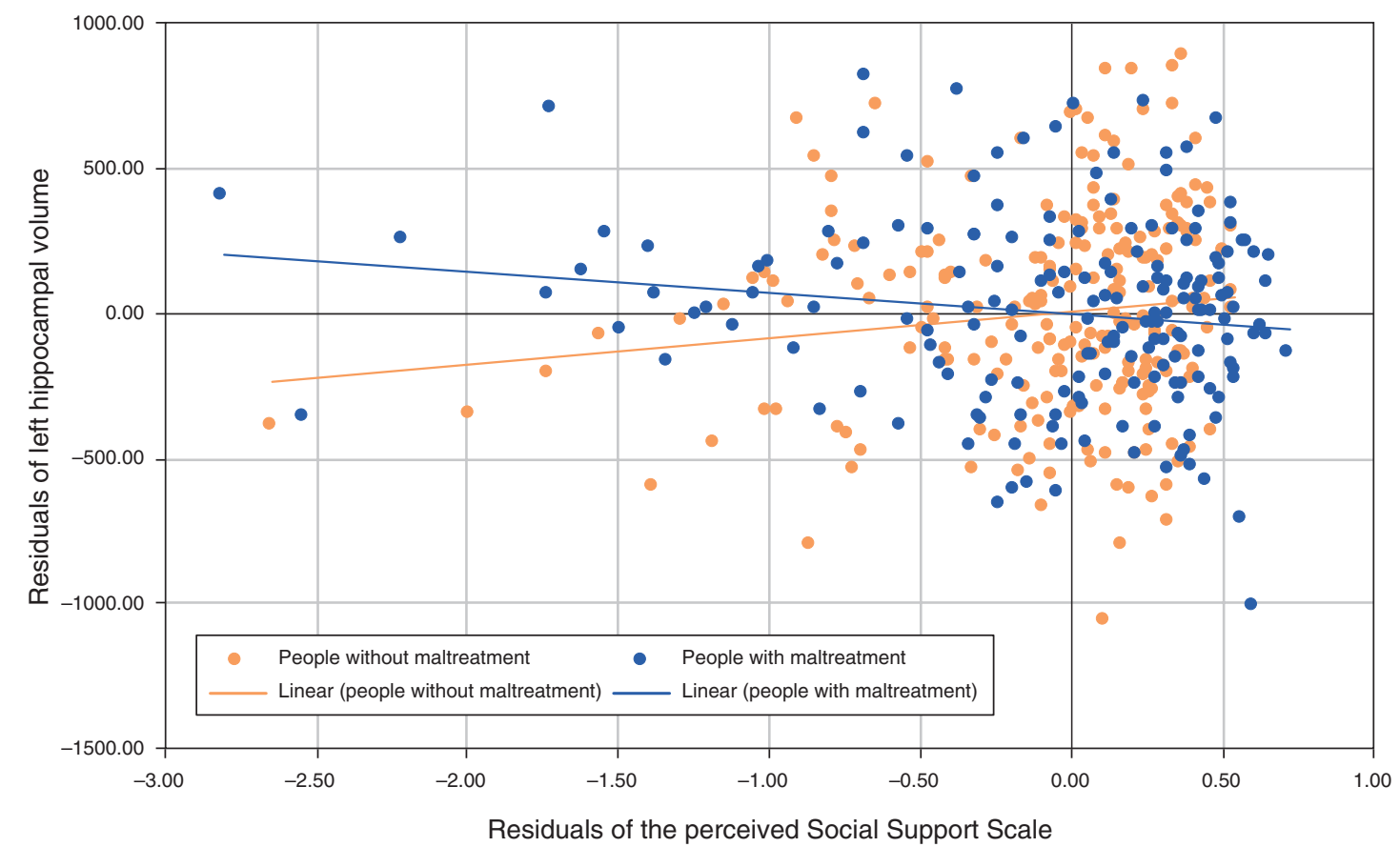

Fig. 2: Scatterplot depicting the relationship between the unstandardized residuals of social support and hippocampal volume in people without maltreatment compared to people with maltreatment. We adjusted the unstandardized residuals for the nuisance regressors of the model (age, sex, total intracranial volume, verbal intelligence, site and body-coil change).

MDD. ${ }^{52}$ To conclude, lower hippocampal volume represents an increased vulnerability toward the impact of stress likely resulting in the development of psychopathology.

The present study focused on social support (a protective factor that has been positively associated with brain volume) and its interaction with childhood maltreatment in the brain structure of the hippocampus. Our results revealed no main effect of social support, but an interaction between social support and childhood maltreatment on hippocampal volume. In people without maltreatment, we found a trend toward a positive association between social support and hippocampal volume. In line with this, previous studies have provided evidence that caregiver support during preschool predicts growth in hippocampal volume in schoolaged children. ${ }^{25,26}$ Our study is the first to show such a trend in adulthood. In adults, previous studies have investigated the relationship between social support and brain volumes outside of the hippocampus; they found a positive association between social support and the volumes of the amygdala, posterior cingulate and lingual cortex. ${ }^{11,27}$ Our study extends the positive association between social support and hippocampal volume that has been found in school-aged and preschool children to adulthood.

In contrast to people without maltreatment, people with maltreatment exhibited a negative association between social support and hippocampal volume. This implies a lower receptiveness of people with maltreatment to social support, resulting in altered brain morphology compared to people without maltreatment. Therefore, our results corroborate the conclusions of a recent longitudinal study, which revealed that later environmental enrichment did not result in increased brain volumes in maltreated children. ${ }^{30}$ In our study, we expected that perceived social support (as a result of positive interpersonal interaction) would be associated with feeling less overwhelmed with problems in everyday life. This was underlined by the "buffering hypothesis," stating that more social support may compensate for the effect of stress on health. ${ }^{16}$ However, previous studies have shown mixed results with respect to a buffering effect of caregiving support during childhood on brain structure in people with maltreatment, ${ }^{23-26}$ depending on the time period of investigation. Specifically, in contrast to evidence for a general positive association between caregiver support and hippocampal volume increases during preschool, Luby and colleagues have shown that positive associations between hippocampal volume and caregiver support are limited in school-aged children to those without previous experience of maltreatment. ${ }^{23}$ Their study does not suggest a buffering effect of support on brain structure in children after preschool. Our study extends this finding to adulthood: our findings did not 
support a buffering effect of social support on brain structure and even generated a hypothesis related to a putative deleterious effect of social support on brain structure in people with maltreatment.

As for the mechanisms involved in this somewhat unexpected finding, it could be speculated that altered perception of social contact in people with maltreatment might play a role. We suggest that social support may result in an increase in stress instead of a reduction in people with maltreatment that is mirrored in a further attrition of brain structure with increased social support. This idea is corroborated by previous studies that have revealed increased amygdala activation to negative facial expressions ${ }^{5}$ in people with maltreatment. Interestingly, the heightened amygdala activation already occurs during the early phases of the neural response to social stimuli, when nonconscious components predominate. ${ }^{53,54}$ This negative processing bias for emotional faces might reduce responsiveness to positive facial stimuli and increase susceptibility to aversive interpersonal stimuli, rendering any kind of social interaction less beneficial or even detrimental at automatic levels of neural processing. This is supported by research revealing attentional and identification biases toward threat-related social information in people with maltreatment. Early studies describe a heightened perceptual sensitivity to anger, ${ }^{55,56}$ even in stimuli containing low emotional intensity of anger. An increased sensitivity to anger might lead to behavioural adaptations that enable an individual to avoid situations that could lead to abuse. However, these adaptations in emotion processing and interpretation likely result in the misinterpretation of benevolent social contact. A recent functional MRI study that investigated social touch revealed further aberrations in social information processing in people with maltreatment: they prefer a greater interpersonal distance and show discomfort with fast touch in contrast to slow touch. ${ }^{57}$ These behavioural alterations have also been linked to exaggerated responses in the right somatosensory and posterior insular cortex. ${ }^{57}$ Severe childhood maltreatment has also been associated with a decreased activation of the hippocampus toward slow touch, and slow touch and hippocampus activation were positively associated in the sample. ${ }^{57}$ This implies that even nonthreatening slow touch is processed differently in people with maltreatment. To sum up, social interaction such as physical or emotional contact with others may result in an increase in stress instead of a reduction in people with maltreatment. Hence, social support might accelerate the attrition of brain structure in people with maltreatment. To substantiate this hypothesis, future research should investigate the neuroendocrine responses of people with maltreatment during the experience of social support, as well as their relationship with brain structural alterations.

Our results also contribute to a further understanding of the development of mental disorders. Research describes childhood maltreatment as an environmental factor that likely results in the development of mental disorders such as depression. ${ }^{58}$ Our findings imply that the detrimental effect of childhood maltreatment affects the association between a protective factor (namely social support) and brain structure.
We assume that childhood maltreatment as an early detrimental social experience strongly affects the perception and processing of social relationships, leading to decreased receptivity to social support. Importantly, we need to bear in mind that we investigated a sample of participants without evidence of psychopathology. To allow for conclusions related to the development of mental disorders, we divided our sample into 2 groups using established CTQ cut-offs: people at risk for mental health disorders because of childhood maltreatment and people without maltreatment. This strategy allowed us to form further hypotheses about the negative relationship between social support and hippocampal volume in people with maltreatment and how this may affect the development of a mental disorder. It also shed light on potential target mechanisms for preventive measures. The association of social support with lower hippocampal volume might indicate an increased vulnerability in social situations, resulting from altered perception of social stimuli and leading to a further attrition of brain structure during increased social support. Importantly, this bias in perception of social stimuli might be subconscious, because it is more pronounced during subliminal presentation of social stimuli such as emotional faces. ${ }^{54}$ Thus, people might report that they feel supported but still exhibit a bias related to social perception. With respect to resilience, this indicates reduced susceptibility to social support as a protective factor in people with maltreatment, implying that people with maltreatment do not show increased resilience to the development of mental disorders when they experience increased social support. This hypothesis may mean that preventive measures aimed at addressing the perception of social stimuli are warranted, such as cognitive bias modification. It might also be helpful to allow for corrective social experiences within the working alliance during prevention.

To our knowledge, this is the first study to investigate how social support moderates the association between maltreatment and hippocampal volume in adulthood. The results suggest a different association between brain structure and protective factors in healthy people who are exposed to maltreatment and at risk for developing mental disorders. However, the cross-sectional nature of our study did not allow for conclusions about the role of our interaction effect in resilience. For example, people exposed to childhood maltreatment who were mentally healthy in our cross-sectional study could develop mental disorders later in life, regardless of their individual hippocampal volume or perceived social support. Our results imply that it is important to consider how protective factors interact with risk factors to further investigate resilience to psychopathology. Because a crosssectional study is only a snapshot of the relationship between risk, protection and brain volume at a single point in time, the role of our interaction effect related to resilience could also be different: the "mismatch hypothesis" notes that people exposed to early adversity express higher vulnerability to mental disorders when they are exposed to lower stress levels-a mismatch in stress exposure during their upbringing and in their adult environment. To reduce mismatch, people with maltreatment and social support can 
expose themselves to higher levels of stress without developing psychopathology. Because higher stress is associated with lower hippocampal volume, ${ }^{59}$ this results in a negative relationship between social support and hippocampal volume in these individuals. A different hypothesis - the "cumulative stress hypothesis" - states that as stress accumulates, people become more vulnerable to mental disorders. Accumulated stress might also result in lower hippocampal volume. ${ }^{19}$ People with lower hippocampal volumes (resembling increased stress) might seek social support to maintain mental health, and people with higher hippocampal volumes (resembling lower stress levels) might not seek increased social support because they are relatively protected. People with maltreatment, at risk because of their lower hippocampal volume and previous adverse life experiences, might seek protective factors to establish resilience. Importantly, we cannot draw conclusions about the relationship between social support and hippocampal volume in clinical populations, because we investigated healthy controls. Future studies should investigate the relationship between social support and brain structure in people with maltreatment using a longitudinal design, to investigate the role of our interaction effect in the development of psychopathology.

To our knowledge, this is the first study to address the question of whether the association between childhood maltreatment and brain structure is moderated by a protective factor during adulthood. The results imply that the association between brain structure and protective factors differs between healthy people at risk for developing mental disorders and healthy people who are not at risk. With respect to resilience, it might be that people with maltreatment are less susceptible to social support as a protective factor and do not show increased resilience to the development of mental disorders when they experience increased social support. Accordingly, there is growing evidence ${ }^{23,30}$ pointing to a need for specific interventions and preventive measures that address social perception in people who have experienced maltreatment. ${ }^{60}$

\section{Limitations}

All results of our study should be considered in the light of some limitations. First, data on childhood maltreatment were acquired using a retrospective self-reporting questionnaire, which implicates the possible creation of negative recall bias. Second, experienced childhood maltreatment might alter perception of social support and lead people with maltreatment to score the Social Support Questionnaire differently. ${ }^{61}$ Further, we measured perceived social support. ${ }^{35}$ The questionnaire collects information about the subjective perception of an available social network and social support in the case of need. In contrast, CTQ scores evaluate events of childhood maltreatment from a retrospective point of view. It might be that social support during the experience of trauma hinders the effect of childhood maltreatment on stress response and regulation, resulting in decreased attrition of hippocampal volume. However, this question could not be addressed by the present study.

\section{Conclusion}

Our study revealed a differential impact of social support on hippocampal volume, dependent on the previous experience of childhood maltreatment. Although people without maltreatment showed higher hippocampal volumes with higher social support, people with maltreatment showed lower hippocampal volumes in response to social support. This study confirms and replicates a detrimental effect of childhood maltreatment on hippocampal volume. Childhood maltreatment appears to moderate the effect of social support on brain structure, potentially hampering protective effects and leading to a decrease in hippocampal volume with higher social support in people with maltreatment. The results point to a need for specific preventive measures for people who have been maltreated that apply to the experience and perception of interpersonal relationships.

Acknowledgements: We acknowledge support from the Open Access Publication Fund of the University of Muenster. We acknowledge the contribution to the present work of all current and former members of the DFG FOR 2107 research group.

Affiliations: From the Department of Psychiatry, University of Münster, Münster, Germany (Förster, Danzer, Redlich, Opel, Grotegerd, Leehr, Dohm, Enneking, Meinert, Goltermann, Lemke, Waltemate, Thiel, Behnert, Hahn, Repple, Dannlowski); the Clinical Psychology and Behavioral Neuroscience, Faculty of Psychology, TU Dresden, Dresden, Germany (Förster); the Department of Clinical Psychology, University of Halle, Halle, Germany (Redlich); the Department of Psychiatry and Psychotherapy, University of Marburg, Marburg, Germany (Brosch, Stein, Meller, Ringwald, Schmitt, Steinsträter, Jansen, Krug, Nenadic, Kircher); the Core-Unit Brain Imaging, Faculty of Medicine, University of Marburg, Marburg, Germany (Jansen); the Department of Psychiatry, University of Bonn, Bonn, Germany (Krug); and the University Clinic for Clinical Radiology, University of Münster, Münster, Germany (Kugel, Heindel).

Funding: This work is part of the German multicenter consortium "Neurobiology of Affective Disorders. A translational perspective on brain structure and function. The present work was funded by the German Research Foundation (DFG, grant FOR2107 DA1151/5-1 and DA1151/5-2 to UD; SFB-TRR58, Projects C09 and Z02 to UD) and the Interdisciplinary Center for Clinical Research (IZKF) of the medical faculty of Münster (grant Dan3/012/17 to U. Dannlowski).

Principal investigators with respective areas of responsibility of the FOR2107 consortium that are relevant to this particular article, WP1, FOR2107/MACS cohort and brain imaging: A. Jansen (JA 1890/7-1, JA 1890/7-2), A. Krug (KR 3822/5-1, KR 3822/7-2), I. Nenadic (NE 2254/1-2; NE 2254/3), T. Kircher (speaker FOR2107; DFG grant numbers KI 588/14-1, KI 588/14-2), T. Hahn (HA 7070/2-2) and U. Dannlowski (co-speaker FOR2107; DA 1151/5-1, DA $1151 / 5-2)$.

Competing interests: None declared.

Contributors: K. Förster, L. Danzer, A. Jansen and U. Dannlowski designed the study. K. Förster, N. Opel, E. Leehr, K. Dohm, V. Enneking, S. Meinert, J. Goltermann, H. Lemke, L. Waltemate, K. Thiel, K. Brosch, F. Stein, T. Meller, K. Ringwald, S. Schmitt, A. Krug, I. Nenadic, H. Kugel and J. Repple acquired the data, which K. Förster, R. Redlich, D. Grotegerd, K. Behnert, O. Steinsträter, T. Kircher, T. Hahn and W. Heindel analyzed. K. Förster, L. Danzer, K. Behnert, O. Steinsträter, T. Kircher and U. Dannlowski wrote the article, which R. Redlich, N. Opel, D. Grotegerd, E. Leehr, K. Dohm, V. Enneking, S. Meinert, J. Goltermann, H. Lemke, L. Waltemate, K. Thiel, K. Brosch, F. Stein, T. Meller, K. Ringwald, S. Schmitt, A. Jansen, A. Krug, 
I. Nenadic, T. Hahn, H. Kugel, W. Heindel and J. Repple reviewed. All authors approved the final version to be published and can certify that no other individuals not listed as authors have made substantial contributions to the paper.

Content licence: This is an Open Access article distributed in accordance with the terms of the Creative Commons Attribution (CC BY-NC-ND 4.0) licence, which permits use, distribution and reproduction in any medium, provided that the original publication is properly cited, the use is noncommercial (i.e., research or educational use), and no modifications or adaptations are made. See: https:/ / creativecommons.org/licenses/by-nc-nd/4.0/

\section{References}

1. Bernet CZ, Stein MB. Relationship of childhood maltreatment to the onset and course of major depression in adulthood. Depress Anxiety 1999;9:169-74.

2. Nemeroff CB. Paradise lost: the neurobiological and clinical consequences of child abuse and neglect. Neuron 2016;89:892-909.

3. Teicher MH, Samson JA, Anderson CM, et al. The effects of childhood maltreatment on brain structure, function and connectivity. Nat Rev Neurosci 2016;17:652-66.

4. Opel N, Redlich R, Zwanzger P, et al. Hippocampal atrophy in major depression: a function of childhood maltreatment rather than diagnosis? Neuropsychopharmacology 2014;39:2723-31.

5. Dannlowski U, Stuhrmann A, Beutelmann V, et al. Limbic scars: long-term consequences of childhood maltreatment revealed by functional and structural magnetic resonance imaging. Biol Psychiatry 2012;71:286-93.

6. Redlich R, Opel N, Bürger C, et al. The limbic system in youth depression: brain structural and functional alterations in adolescent in-patients with severe depression. Neuropsychopharmacology 2018;43:546-54.

7. Opel N, Redlich R, Dohm K, et al. Mediation of the influence of childhood maltreatment on depression relapse by cortical structure: a 2-year longitudinal observational study. Lancet Psychiatry 2019;6:318-26

8. Rao U, Chen LA, Bidesi AS, et al. Hippocampal changes associated with early-life adversity and vulnerability to depression. Biol Psychiatry 2010;67:357-64

9. Herringa RJ, Birn RM, Ruttle PL, et al. Childhood maltreatment is associated with altered fear circuitry and increased internalizing symptoms by late adolescence. Proc Natl Acad Sci U S A 2013; 110:19119-24.

10. Birn RM, Patriat R, Phillips ML, et al. Childhood maltreatment and combat posttraumatic stress differentially predict fear-related fronto-subcortical connectivity. Depress Anxiety 2014;31:880-92.

11. Sato W, Kochiyama T, Kubota Y, et al. The association between perceived social support and amygdala structure. Neuropsychologia 2016;85:237-44.

12. Dufour MH, Nadeau L, Bertrand K. Les facteurs de resilience chez les victimes d'abus sexuel: état de la question. Child Abuse Negl 2000;24:781-97.

13. Cohen S, Hoberman HM. Positive events and social supports as buffers of life change stress. J Appl Soc Psychol 1983;13:99-125.

14. Cobb S. Social support as a moderator of life stress. Psychosom Med 1976;38:300-14.

15. Dunkel D, Antretter E, Fröhlich-Walser S, et al. Evaluation of the short-form social support questionnaire (FSozU-K-22) in clinical and non-clinical samples. Psychother Psychosom Med Psychol 2005;55:266-77.

16. Cohen S, Wills TA. Stress, social support and the buffering hypothesis. Psychol Bull 1985;98:310-57.

17. Lakey B. Perceived social support and happiness: the role of personality and relational processes. In: Boniwell I, David S, Conley Ayers A, editors. Oxford handbook of happiness. Oxford: Oxford University Press; 2013. 1-16.

18. Meng X, Fleury MJ, Xiang YT, et al. Resilience and protective factors among people with a history of child maltreatment: a systematic review. Soc Psychiatry Psychiatr Epidemiol 2018:53:453-75.

19. Sapolsky R, Krey LC, Mcewen BS. Glucocorticoid-sensitive hippocampal neurons are involved in terminating the adrenocortical stress response. Proc Natl Acad Sci U S A 1984;81:6174-7.
20. Utsunomiya H, Takano K, Okazaki M, et al. Development of the temporal lobe in infants and children: analysis by MR-based volumetry. AJNR J Neuroradiol 1999;20:717-23.

21. Sorrells SF, Paredes MF, Cebrian-Silla A, et al. Human hippocampal neurogenesis drops sharply in children to undetectable levels in adults. Nature 2018;555:377-81.

22. Rao H, Betancourt L, Giannetta JM, et al. Early parental care is important for hippocampal maturation: evidence from brain morphology in humans. Neuroimage Elsevier Inc. 2010;49:1144-50.

23. Luby JL, Tillman R, Barch DM. Association of timing of adverse childhood experiences and caregiver support with regionally specific brain development in adolescents. JAMA Netw Open 2019; 2:e1911426.

24. Luby JL. Treatment of anxiety and depression in the preschool period. J Am Acad Child Adolesc Psychiatry 2013;52:346-58.

25. Luby JL, Barch DM, Belden A, et al. Maternal support in early childhood predicts larger hippocampal volumes at school age. Proc Natl Acad Sci U S A 2012;109:2854-9.

26. Luby JL, Belden A, Harms MP, et al. Preschool is a sensitive period for the influence of maternal support on the trajectory of hippocampal development. Proc Natl Acad Sci U S A 2016;113:5742-7.

27. Che X, Wei DT, Li WF, et al. The correlation between gray matter volume and perceived social support: a voxel-based morphometry study. Soc Neurosci 2014;9:152-9.

28. Hyde LW, Gorka A, Manuck SB, et al. Perceived social support moderates the link between threat-related amygdala reactivity and trait anxiety. Neuropsychologia 2011;49:651-6.

29. Hager AD, Runtz MG. Physical and psychological maltreatment in childhood and later health problems in women: an exploratory investigation of the roles of perceived stress and coping strategies. Child Abuse Negl 2012;36:393-403.

30. Mackes NK, Golm D, Sarkar S, et al. Early childhood deprivation is associated with alterations in adult brain structure despite subsequent environmental enrichment. Proc Natl Acad Sci U S A 2020; 117:641-9.

31. Kircher T, Wöhr M, Nenadic I, et al. Neurobiology of the major psychoses: a translational perspective on brain structure and function-the FOR2107 consortium. Eur Arch Psychiatry Clin Neurosci 2019;269:949-62

32. Wittchen H-U, Wunderlich U, Gruschwitz S, et al. SKID-I. Strukturiertes Klinisches Interview für DSM-IV. Göttingen, Germany: Hogrefe; 1997.

33. Walker EA, Gelfand A, Katon WJ, et al. Adult health status of women with histories of childhood abuse and neglect. Am J Med 1999;107:332-9.

34. Wingenfeld K, Spitzer C, Mensebach C, et al. The German version of the Childhood Trauma Questionnaire (CTQ): preliminary psychometric properties. Psychother Psychosom Med Psychol 2010;60:442-50.

35. Fydrich T, Geyer M, Hessel A, et al. Social Support Questionnaire (F-SozU): norms of a representative sample. Diagnostica 1999;45:212-6.

36. Fydrich T, Sommer G, Menzel U, et al. Fragebogen zur sozialen Unterstützung (Kurzform; SozU-K-22). Z Klin Psychol 1987;16:434-6.

37. Bernstein DP, Stein JA, Newcomb MD, et al. Development and validation of a brief screening version of the Childhood Trauma Questionnaire. Child Abuse Negl 2003;27:169-90.

38. Bernstein DP, Fink L, Handelsman L, et al. Initial reliability and validity of a new retrospective measure of child abuse and neglect. Am J Psychiatry 1994;151:1132-6.

39. Beck AT, Steer R, Brown G. Beck depression inventory manual. Arch Gen Psychiatry 1987;4:561-71.

40. Lehrl S. Mehrfachwahl-Wortschatz-Intelligenzest (MWT-B). 5th ed. Balingen, Germany: Spitta-Verlag; 2005.

41. Satzger W, Fessmann H, Engel RR. Liefern HAWIE-R, WST und MWT-B vergleichbare IQ-Werte? Z Differ Diagn Psychol 2002; 23:159-70.

42. Vogelbacher C, Möbius TWD, Sommer J, et al. The MarburgMünster Affective Disorders Cohort Study (MACS): a quality assurance protocol for MR neuroimaging data. Neuroimage 2018; 172:450-60.

43. Desikan RS, Ségonne F, Fischl B, et al. An automated labeling system for subdividing the human cerebral cortex on MRI scans into gyral based regions of interest. Neuroimage 2006;31:968-80.

44. Teicher MH, Andersen SL, Polcari A, et al. The neurobiological consequences of early stress and childhood maltreatment. Neurosci Biobehav Rev 2003;27:33-44 
45. MacQueen G, Frodl T. The hippocampus in major depression: evidence for the convergence of the bench and bedside in psychiatric research. Mol Psychiatry 2011;16:252-64.

46. Yehuda R, Golier JA, Tischler L, et al. Hippocampal volume in aging combat veterans with and without post-traumatic stress disorder: relation to risk and resilience factors. I Psychiatr Res 2007; 41:435-45.

47. Amico F, Meisenzahl E, Koutsouleris N, et al. Structural MRI correlates for vulnerability and resilience to major depressive disorder. J Psychiatry Neurosci 2011;36:15-22.

48. Sapolsky RM. The possibility of neurotoxicity in the hippocampus in major depression: a primer on neuron death. Biol Psychiatry 2000;48:755-65.

49. Lyall AE, Feng S, Yiujuan G, et al. Dynamic development of regional cortical thickness and surface area in early childhood. Cereb Cortex 2015;25:2204-12.

50. Lupien SJ, McEwen BS, Gunnar MR, et al. Effects of stress throughout the lifespan on the brain, behaviour and cognition. Nat Rev Neurosci 2009;10:434-45.

51. Bangalore L. Brain development. New York: Infobase Publishing; 2009.

52. Zaremba D, Enneking V, Meinert S, et al. Effects of cumulative illness severity on hippocampal gray matter volume in major depression: a voxel-based morphometry study. Psychol Med 2018; 48:2391-8.
53. Garrett AS, Carrion V, Kletter H, et al. Brain activation to facial expressions in youth with PTSD symptoms. Depress Anxiety 2012;29:449-59.

54. Dannlowski U, Kugel H, Huber F, et al. Childhood maltreatment is associated with an automatic negative emotion processing bias in the amygdala. Hum Brain Mapp 2013;34:2899-909.

55. Pollak SD, Tolley-Schell SA. Selective attention to facial emotion in physically abused children. J Abnorm Psychol 2003;112:323-38.

56. Gibb BE, Schofield CA, Coles ME. Reported history of childhood abuse and young adults' information-processing biases for facial displays of emotion. Child Maltreat 2009;14:148-56.

57. Maier A, Gieling C, Heinen-Ludwig L, et al. Association of childhood maltreatment with interpersonal distance and social touch preferences in adulthood. Am J Psychiatry 2020;177:37-46.

58. Teicher $\mathrm{MH}$, Samson JA. Annual research review: enduring neurobiological effects of childhood abuse and neglect. J Child Psychol Psychiatry 2016;57:241-66.

59. McEwen BS, Gould EA, Sakai RR. The vulnerability of the hippocampus to protective and destructive effects of glucocorticoids in relation to stress. Br J Psychiatry Suppl 1992;160:18-23.

60. McLaughlin KA, DeCross SN, Jovanovic T, et al. Mechanisms linking childhood adversity with psychopathology: learning as an intervention target. Behav Res Ther 2019;118:101-9.

61. Pepin EN, Banyard VL. Social support: a mediator between child maltreatment and developmental outcomes. J Youth Adolesc 2006;35:617-30. 